\title{
miR-155 induced transcriptome changes in the MCF-7 breast cancer cell line leads to enhanced mitogen activated protein kinase signaling
}

\author{
Elizabeth C. Martin ${ }^{1}$, Adrienne E. Krebs ${ }^{1}$, Hope E. Burks ${ }^{1}$, Steven Elliott ${ }^{1}$, Melody \\ Baddoo $^{2,3}$, Bridgette M. Collins-Burow ${ }^{1}$, Erik K. Flemington ${ }^{2,3}$ and Matthew E. \\ Burow ${ }^{1,2,4}$ \\ ${ }^{1}$ Department of Medicine-Section of Hematology and Medical Oncology, Tulane University, New Orleans, LA \\ ${ }^{2}$ Tulane Cancer Center, Tulane University, New Orleans, LA \\ ${ }^{3}$ Department of Pathology, Tulane University, New Orleans, LA \\ ${ }^{4}$ Department of Pharmacology, Tulane University, New Orleans, LA \\ Correspondence to: Matthew E. Burow, email: mburow@tulane.edu
}

Keywords: microRNA-155, breast cancer, MAPK, p38, 3'UTR, RNA-seq

Received: September 04, $2014 \quad$ Accepted: October 01, $2014 \quad$ Published: October 01, 2014

This is an open-access article distributed under the terms of the Creative Commons Attribution License, which permits unrestricted use, distribution, and reproduction in any medium, provided the original author and source are credited.

\section{ABSTRACT}

A single microRNA (miRNA) has the potential to regulate thousands of genes and thus govern multiple signaling pathways at once. miR-155 is an oncogenic miRNA which regulates many cellular pathways, designating it as a multifaceted regulator of proliferation, chemo-resistance, and apoptosis. While many singular targeted effects of miR-155 have been defined and an oncogenic role has been attributed to $\mathrm{miR-155}$ expression, the global effect of miR-155 on the cellular transcriptomes of an $\mathrm{ER}^{+}$breast cancer cell line has yet to be determined. Here we demonstrate that miR-155 expression increases tumorigenesis in vivo and we determine miR155 mediated transcriptome changes through next generation sequencing analysis. miR-155 expression alters many signaling pathways, with the chief altered pathway being the MAPK signaling cascade and miR-155 induces shortening of target mRNA $3^{\prime}$ UTRs and alternative isoform expression of MAPK related genes. In addition there is an observed increase in protein phosphorylation of components of MAPK signaling including ERK1/ 2 and AP-1 complex members (Fra-1 and c-Fos) as well as elevated gene expression of MAPK regulated genes Zeb1, Snail, Plaur, and SerpinE1.

\section{INTRODUCTION}

microRNAs (miRNAs) are small non-coding RNAs known to mediate cancer progression [1-4]. Among cancers of the breast, expression profiling of miRNAs shows correlations between miRNA expression and receptor status for estrogen receptor (ER), progesterone receptor (PgR), and V-Erb-B2 avian erythroblastic leukemia viral oncogene homolog 2 (HER2/Nue) $[5,6]$. In addition to a prognostic correlation, miRNA expression correlates with all facets of breast cancer progression including proliferation, endocrine resistance, and metastasis [7]. One miRNA, microRNA-155 (miR-155) is known to regulate multiple aspects of breast cancer progression in addition to demonstrating a correlation with loss of receptor status [2, 3, 8, 9]. Located within the noncoding $\mathrm{B}$ cell integration cluster (BIC) gene, microRNA-155 (miR-155) was first discovered through retroviral integrations in B cell lymphomas [12]. Currently miR-155 is found to be amplified in many cancers, irrespective of retroviral integration [13]. miR-155 expression is such a powerful mutagen that transgenic mice over-expressing this single miRNA in B-cells demonstrate an accumulation of pre-B cells and eventually develop B-cell malignancies lymphoma/leukemia [14]. As one of the more highly evaluated miRNAs, many targets are known and miR-155 is an established oncogenic miRNA $[2,3,15]$. Through targeting of genes such as SOCS1, TP53BP1, and FOXO3a miR-155 is able to enhance cellular proliferation and survival of breast cancer 
cells $[2,10,11]$.

miR-155 is known to regulate cellular proliferation and survival through multiple mechanisms, including the mitogen activated protein kinases (MAPKs) signaling pathways. miR-155 is a positive multi-faceted regulator of MAPK signaling, intervening at heterogeneous points along the signaling cascade to enhance signaling. Through targeting of inositol polyphosphate-5-phosphatase (SHIP1) miR-155 enhances extracellular signal-regulated kinases (ERK) activation [17]. In a glioblastoma cell line, miR-155 expression enhanced ERK phosphorylation and cellular proliferation while alternatively, the loss of miR155 expression led to decreased ERK activation in B-cells $[18,19]$. Downstream MAPK effectors such as AP-1 targeting of DET1. Targeting of DET1 stabilizes c-Jun transcripts and subsequently increases AP-1 activity [20].

Here we demonstrate through next generation sequencing analysis, global changes in both MAPK ERK1/2 and p38 gene expression due to overexpression of miR-155. In addition, miR-155 enhanced MAPK signaling which was observed through increased MAPK and effector protein phosphorylation.

\section{Over-Expression of miR-155 Enhances Tumorigenesis in vivo}

miR-155 has previously been defined as an oncogenic miRNA enhancing proliferation and survival $[2,3,40,41]$. To observe a comprehensive effect of miR155 over-expression on $\mathrm{ER}^{+}$breast cancer tumorigenesis, MCF-7 cells over-expressing miR-155 (MCF-7-miR-155) were generated and validated as previously described [52]. Cells were then inoculated in ovariectomized CB-17/SCID

A

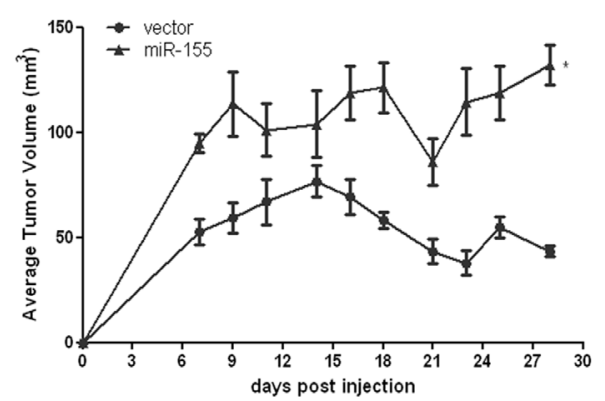
complex member c-Jun, are enhanced through miR-155

\section{RESULTS} female mice in the mammary fat pad (MFP). At necropsy

(day 28 post cell injection), final tumor volume and weight was greater for MCF-7-miR-155 tumors compared to vector tumors (Figure $1 \mathrm{~A}$ and $1 \mathrm{~B}$ respectively), demonstrating that miR-155 expression enhances basal tumorigenesis in vivo.

\section{3'UTR Shortening Leads to Increased Expression of miR-155 MAPK Target Genes}

To determine an underlying mechanism for the observed miR-155 induced tumorigenesis in vivo, MCF7-miR-155 and-vector cell lines were analyzed with next generation sequencing. Subsequently, all miR-155 seed sites, identified through our in-house Seedfinder program, were analyzed. Interestingly, while many predicted miR-155 targets demonstrated an overall repression of expression, having fold expression levels of 0.5 or lower compared to the vector cell line, some predicted targets of miR-155 demonstrated an increase in expression levels (fold $>1.5$ ) (Figure 2A). This increase in expression was observed in miR-155 predicted targets irrespective of whether or not the seed site was an 8-mer or 7-mer site (Figure 2B and 2C respectively). To determine the possible cause for increased expression of mRNAs containing a miR-155 predicted target sites we examined all miR155 predicted targets which contained an 8-mer-seed site and had an increase in expression over 1.5 fold. Of the thirty targets showing increased expression, fourteen of these targets demonstrated very low reads (below 10) in both the MCF-7-vector and -miR-155 cell line and were therefore not chosen for further analysis. Increased cellular proliferation as well as exogenous stimulation can be attributed to shortening of 3'UTR [42, 43]. This has previously been demonstrated in miR-155 overexpressing cell lines, where miR-155 target site was lost due to 3'UTR shorting [44]. As our miR-155 cell line showed increased tumorigenesis, we next sought to explore the possibility that variability of 3'UTR and loss of miR-

B

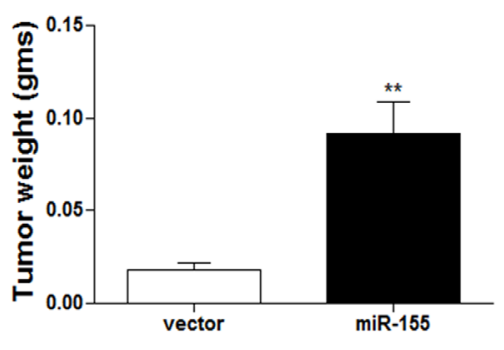

Figure 1: miR-155 Expression Enhances Tumorigenesis in vivo ovariectomized SCID/CB-17 female mice injected bilaterally with 5X10 ${ }^{6}$ MCF-7-vector cells or MCF-7-miR-155 cells, $n=5$ animals/group. (A) Tumor volume, points represent average tumor volume \pm SEM starting at day 7 post injection, measurements were carried out until necroscopy (day 28). (B) final tumor weight at necroscopy (day 28). * significantly different $\mathrm{p}<0.05,{ }^{* *}$ significantly different $\mathrm{p}<0.01$ 
Table 1: Loss of 3'UTR in Genes with miR-155 8-mer Seed Sites

\begin{tabular}{|c|c|c|}
\hline Gene & $\begin{array}{c}\text { MCF-7-miR-155 } \\
\text { 3'UTR Shortening }\end{array}$ & $\begin{array}{c}\text { Relative Expression } \\
\text { miR-155/vector }\end{array}$ \\
\hline SPIN2B & Yes & 1.516575 \\
\hline GLT25D1 & No & 1.557417 \\
\hline MAP3K14 & Yes & 1.849516 \\
\hline CARS2 & Yes & 1.862035 \\
\hline ARVCF & Yes & 2.292287 \\
\hline AGTRAP & Yes & 2.816578 \\
\hline MYLK & No & 3.222538 \\
\hline CARD10 & Yes & 3.237354 \\
\hline IRF2BP2 & Yes & 3.330693 \\
\hline CSNK1G2 & No & 3.472894 \\
\hline TRMT61A & Yes & 3.627442 \\
\hline MAP3K10 & Yes & 3.813621 \\
\hline CEBPB & Yes & 4.871474 \\
\hline PRKAR1B & Yes & 7.552545 \\
\hline SOCS1 & No & 8.080313 \\
\hline SPOCK1 & No & 20.33727 \\
\hline
\end{tabular}

A

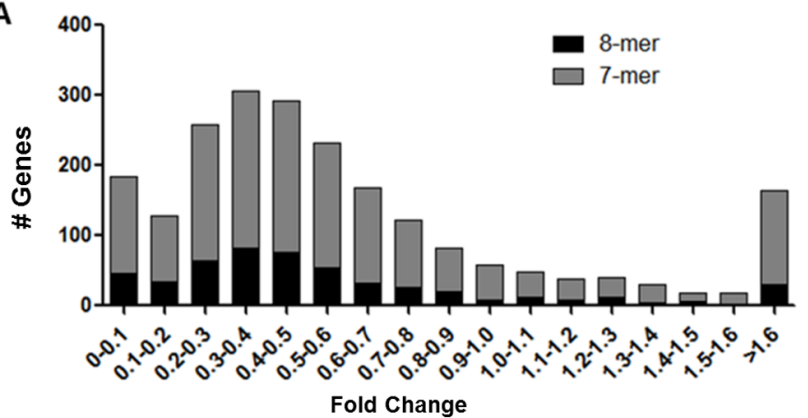

C

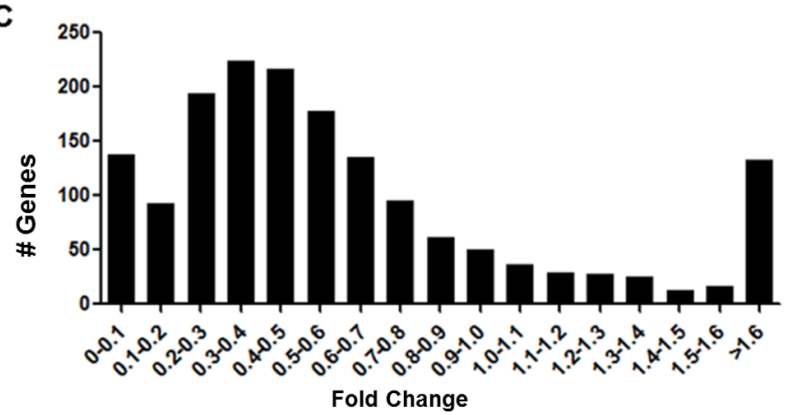

155 target sites occurred in our miR-155 cell line. Of the remaining sixteen targets, ten transcripts with isoforms demonstrating sufficient levels of expression, showed a loss of 3'UTR expression at the miR-155 seed site in the MCF-7-miR-155 cell line compared to -vector (Table 1). One miR-155 target, MAP3K10 demonstrated loss of 3 'UTR in both the vector and miR-155 cell lines (Figure 3A).

Due to the fluid nature of miR-155 over-expression and mRNA target selection, we next used the online pathway interaction database (PID) to determine the top pathways predicted to be targeted by miR-155 through BioCarta derived analysis [http://www.cancer.gov]. Genes which contain a miR-155 seedsite were loaded into the pathway data base to determine if a correlation existed between genes containing a miR-155 seed site and a specific pathway. Relevant pathways were determined based on the number of associated proteins and the relative affinity each protein had for a particular pathway and a $p$ value was assigned to each pathway to demonstrate significance of correlation, the top pathway contained the greatest significant of correlation. The results from this analysis showed that many of the top pathways predicted to be targeted by $\mathrm{miR}-155$ were pathways regulated by growth factors and the cell cycle (Table 2). In addition to miR-155 predicted targets we analyzed all genes that were suppressed by miR-155 over-expression and all genes demonstrating an increase in expression (Table 2). Interestingly, pathway predictions based on down-

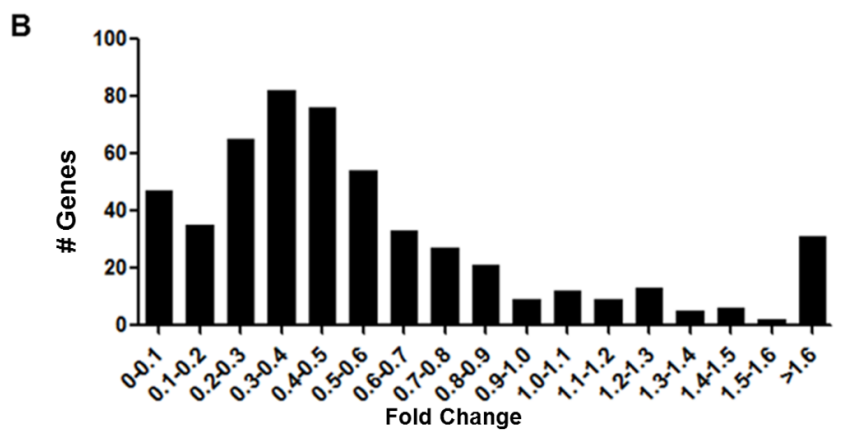

Figure 2: Next Generation Sequencing Analysis of miR-155 in the MCF-7-miR-155 Cell Line MCF-7-vector and -miR155 cell lines were extracted for total RNA and were analyzed through next generation sequencing. Genes containing any miR-155 seed site were pooled and further analyzed. Results represent fold change of predicted miR-155 targets with (A) $8 \mathrm{mer}$ or $7 \mathrm{mer}$ site (B) 8 mer site (C) 7mer site. X-axis depicts fold change in change expression between MCF-7-miR-155 cell line and vector, y-axis demonstrates number of genes. 
Table 2: Analysis of miR-155 Regulated Pathways

\begin{tabular}{|c|c|c|}
\hline Up Regulated Pathways & Down Regulated Pathways & miR-155 Targeted Pathways \\
\hline MAPK Signaling Pathway & Rho cell motility & Rac1 cell motility signaling pathway \\
\hline RHO cell motility signaling pathway & Rac1 cell motility signaling pathway & Regulation of bad phosphorylation \\
\hline Integrin signaling pathway & Ras signaling pathway & $\begin{array}{c}\text { Bioactive peptide induced signaling } \\
\text { pathway }\end{array}$ \\
\hline Rac1 Cell Motility Signaling Pathway & Integrin signaling pathway & $\begin{array}{c}\text { Transcription factor creb and its } \\
\text { extracellular signals }\end{array}$ \\
\hline $\begin{array}{c}\text { Inhibition of Cellular Proliferation by } \\
\text { Gleevec }\end{array}$ & $\begin{array}{c}\text { Role of Brca1 Brca2 and Atr in cancer } \\
\text { susceptibility }\end{array}$ & Growth hormone signaling pathway \\
\hline $\begin{array}{c}\text { ERK and PI3K collagen in corneal } \\
\text { epithelia }\end{array}$ & Proteasome complex & Rho cell motility signaling pathway \\
\hline Negative effectors of Fas and TNF & $\begin{array}{c}\text { Hiv-1 nef: negative effector of fas and } \\
\text { tnf }\end{array}$ & Cell cycle: $\mathrm{g} 1 / \mathrm{s}$ check point \\
\hline Bioactive $\begin{array}{c}\text { peptide induced signaling } \\
\text { pathway }\end{array}$ & Cyclin E destruction pathway & $\begin{array}{c}\text { Co-stimulatory signal during t-cell } \\
\text { activation }\end{array}$ \\
\hline P38 MAPK signaling pathway & Ifn alph & Igf-1 signaling pathway \\
\hline $\begin{array}{c}\text { Induction of apoptosis through DR3 and } \\
\text { DR4/5 death receptors }\end{array}$ & Map kinase signaling pathway & Ras signaling pathway \\
\hline $\begin{array}{l}\text { Nuclear receptors coordinate the activities } \\
\text { of chromatin remodeling complexes and } \\
\text { coactivators to facilitate initiation of } \\
\text { transcription in carcinoma cells }\end{array}$ & $\begin{array}{l}\text { ERK and PI3 kinase are necessary for } \\
\text { collagen binding in corneal epithelia }\end{array}$ & Phospholipase c signaling pathway \\
\hline Ras signaling 1 & $\begin{array}{r}\text { Hypoxia and p5 } \\
\text { sys }\end{array}$ & $\begin{array}{c}\text { Hiv-1 nef: negative effector of fas and } \\
\text { tnf }\end{array}$ \\
\hline $\begin{array}{l}\text { Control of skeletal myogenesis by HDAC } \\
\text { and calcium/calmodulin dependent kinase } \\
\text { (CAMK) }\end{array}$ & Caspase cascade in apoptosis & $\begin{array}{c}\text { Human cytomegalovirus and map } \\
\text { kinase pathways }\end{array}$ \\
\hline Growth hormone signaling pathway & $\begin{array}{c}\mathrm{Rb} \text { tumor suppressor/check } \\
\text { signaling in response to dna dam }\end{array}$ & Akt signaling pathway \\
\hline ERK1/ERK2 MAPK signaling pathway & D4GDI signaling pathway & Egf signaling pathway \\
\hline IL-7 signal transduction & Cell cycle: g1/s check point ATM & $\begin{array}{l}\text { Multiple antiapoptotic pathways } \\
\text { from igf-1r signaling lead to bad } \\
\text { phosphorylation }\end{array}$ \\
\hline $\begin{array}{c}\text { FC epsilon receptor I signaling in mast } \\
\text { cells }\end{array}$ & Links between pyk 2 and map kinases & Trka receptor signaling pathway \\
\hline IL-22 soluble receptor signaling pathway & $\begin{array}{c}\text { Transcription factor creb and its } \\
\text { extracellular signals }\end{array}$ & Map kinase signaling pathway \\
\hline $\begin{array}{c}\text { Apoptotic DNA-fragmentation and tissue } \\
\text { homeostasis }\end{array}$ & $\begin{array}{c}\text { CDC25 and chk1 regulatory pathway } \\
\text { in response to dna damage }\end{array}$ & $\begin{array}{c}\text { Role of pi3k subunit p85 in regulation } \\
\text { of actin organization and cell }\end{array}$ \\
\hline
\end{tabular}

regulated genes corresponded to MAPK signaling and cell death. Of genes demonstrating increased levels of expression, many were associated with proliferation with the top pathway being MAPK signaling and subsequent MAPK related pathways (p38 MAPK signaling, ERK1/2 MAPK signaling pathway, RAS signaling pathway). As miR-155 has predicted targets of MAPK signaling which would both activate and repress the pathway, we next sought to validate MAPK associated miR-155 predicted targets. Both activators (RSK2, k-RAS, KSR1, FADD, and RAC1) and repressors (PP2AC, DUSP7, DUSP14, and PEA-15) were chosen for further analysis with qPCR.
Many miR-155 MAPK associated predicted targets which are repressors of MAPK signaling showed no significant change in either direction (Figure 4A) while activators of MAPK pathway were either unchanged or demonstrated significant increases in expression levels (Figure 4B). To determine a mechanism for the changes observed in MAPK targets which would enhance MAPK signaling, the 3'UTRs of MAPK activators RAC1, RSK2, k-RAS, and KSR1 were investigated. Only RAC1 and MAP3K10 demonstrate 3'UTR shortening and loss of miR-155 targeting (Table 3). RSK2 was the only predicted miR-155 target demonstrating a significant increase in expression 
A

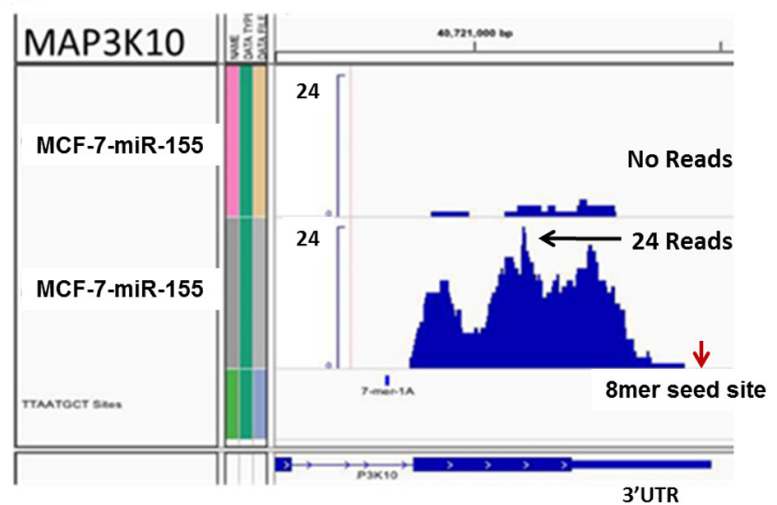

B

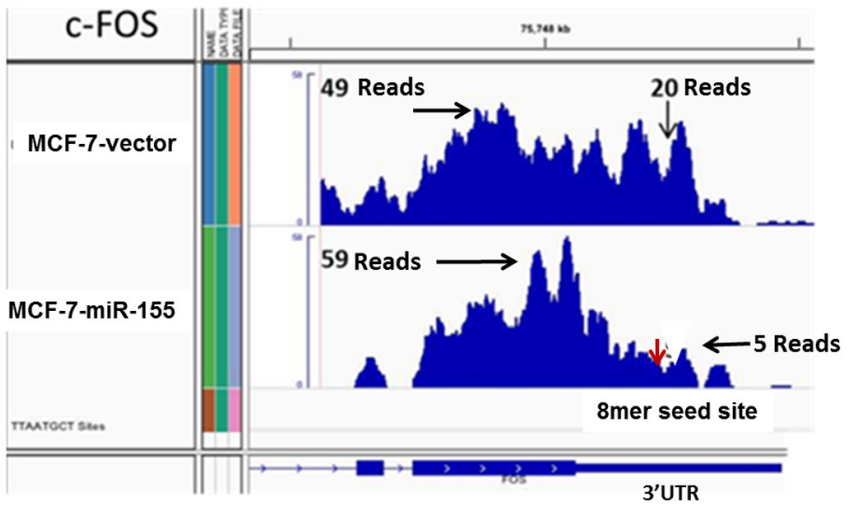

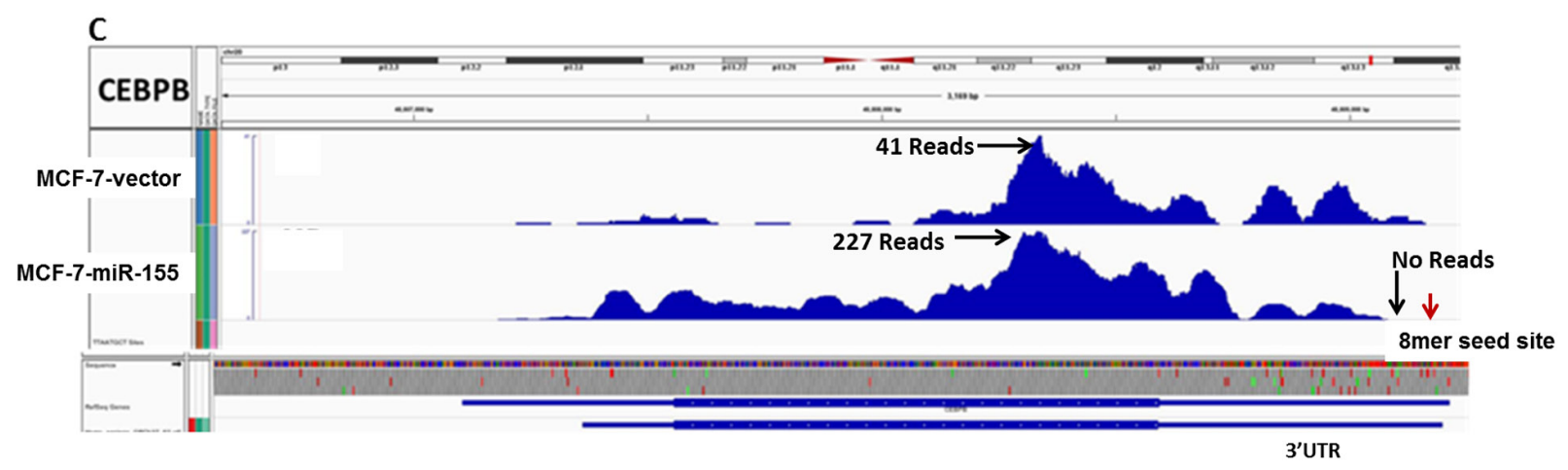

Figure 3: Genes Containing miR-155 Seed Site Demonstrate Loss of 3'UTR in the MCF-7-miR-155 Cell Line. Next generation sequencing analysis, representative pictures of 3'UTR of MAPK associated genes with miR-155 seed site (A) MAP3K10 (B) c-FOS and (C) CEBP $\beta$. Results represent raw reads and reference genome is HG19

A

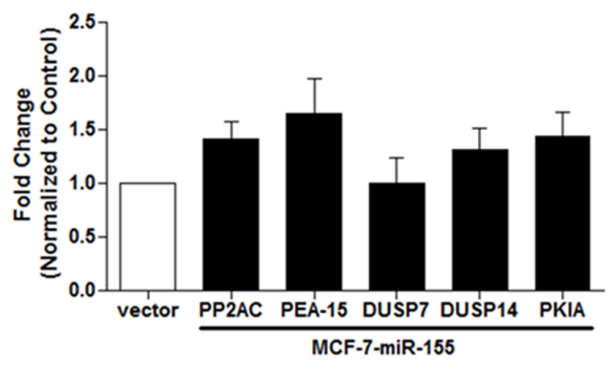

C

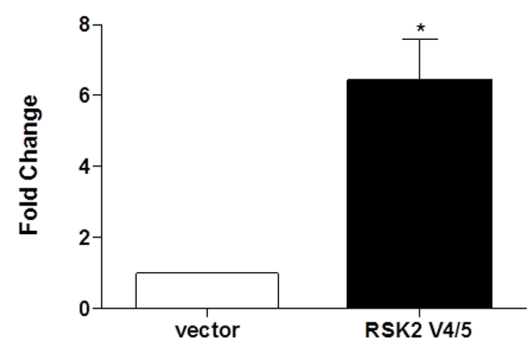

B

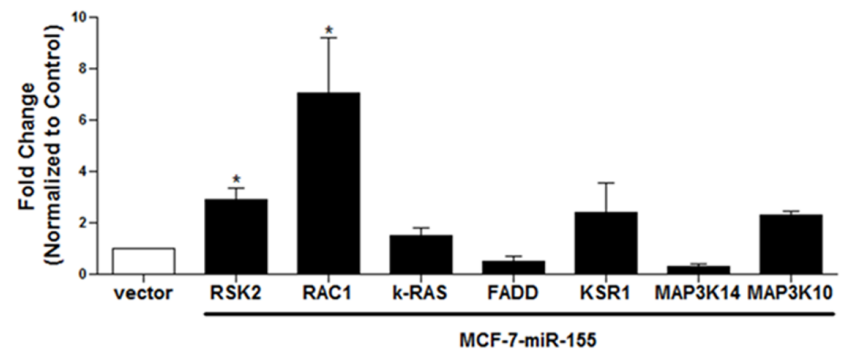

Figure 4: Expression of MAPK Associated Genes with miR-155 Seed Sites Following miR-155 Over-Expression in MCF-7 Cells. MCF-7-vector and MCF-7-miR-155 cells were harvested for qPCR for genes which contain a miR-155 seed site. Genes were selected that (A) repress MAPK signaling (PP2AC, PEA-15, DUSP7, DUSP14, PKIA) and (B) are associated with activated MAPK signaling (RSK2, RAC1, k-RAS, FADD, KSR1, MAP3K14, MAP3K10) (C) qPCR for MCF-7-miR-155 cell line expression of RSK2 isoform variant 4 and 5. Normalization was to actin and MCF-7-vector cells designated as 1. Error bars represent SEM. * $\mathrm{p}<0.05 . \mathrm{n}=3$ 
Table 3: Genes with miR-155 Seed site and of Loss of 3'UTR

\begin{tabular}{|l|l|l|l|}
\hline Gene & 7mer sites & 8mer sites & $\begin{array}{l}\text { 3’UTR } \\
\text { shortening }\end{array}$ \\
\hline RSK2 & 1 & 0 & No \\
\hline Rac1 & 1 & 0 & Yes \\
\hline k-Ras & 3 & 0 & No \\
\hline KSR1 & 0 & 1 & No \\
\hline MAP3K14 & 0 & 1 & No \\
\hline MAP3K10 & 0 & 1 & Yes \\
\hline
\end{tabular}

without a subsequent loss of 3'UTR; we sought to further evaluate the mechanisms responsible for the increase in RSK2 expression. Isoform variance is known to exist among gene transcripts and is known to result in loss of miRNA targeting through altered isoform expression
[44]. qPCR was performed for the RKS2 isoform variants 4 and 5 which demonstrate a truncated transcript and do not contain the same 3'UTR as the other RSK2 transcripts. A significant increase in expression of isoforms $4 / 5$ of RSK2 is observed in the miR-155 over-expressing cell line (Figure 4C). Together, these data indicate that mediators of MAPK signaling are increased in the MCF-7-miR-155 cell line

\section{miR-155 Expression Enhances MAPK Signaling in MCF-7 Breast Cancer Cell Line}

To better assess the global effect of miR-155 on MAPK signaling, all MAPK genes demonstrating detectable expression levels were pooled from next generation sequencing data and analyzed. MAPK signaling is multifaceted, including four major signaling families,
A

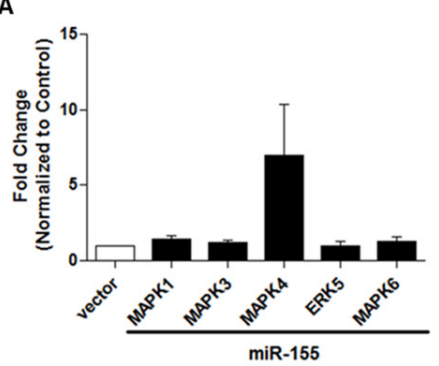

C

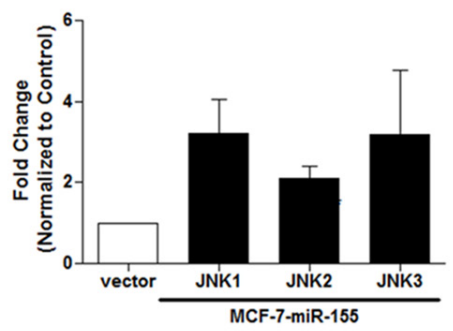

E

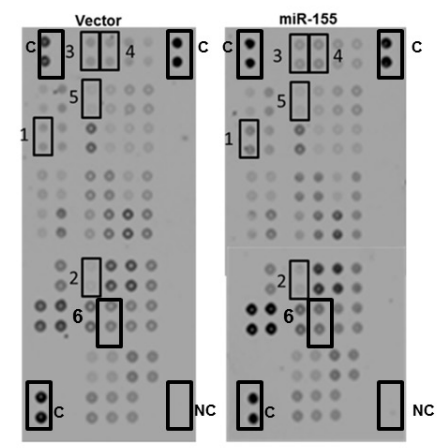

B

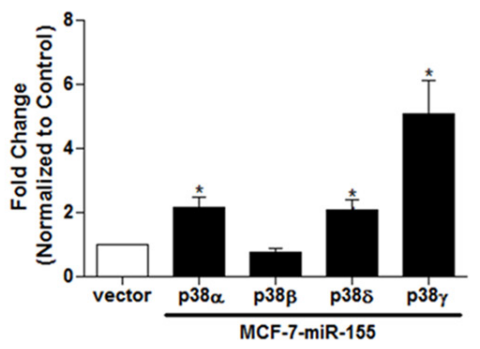

D



F

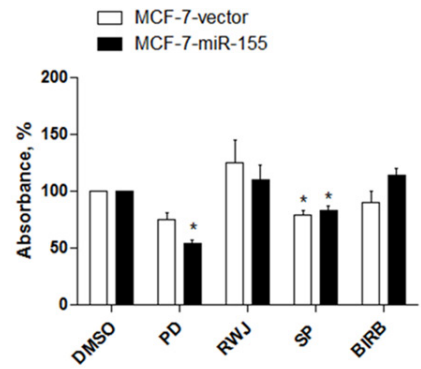

Figure 5: miR-155 Alters MAPK Signaling. MCF-7-vector and -miR-155 cell lines were harvested for qPCR to determine basal expression levels for (A) MAPKs 1-6, (B) p38 isoforms (C) JNK1-3 and (D) MAPK associated genes. Normalization was to actin and vector cells designated as one. Error bars represent SEM, n > 3. (E) Human Phospho-Kinase Protein Array for miR-155 Targets. Representative membranes from 2 independent experiments evaluating a broad range of phosphorylation targets for MCF-7 Vector and miR-155 cell lines. Selected targets are ERK1/2 T202 Y204 (1), p70 S6 Kinase T389 (2), Src Y419 (3), mTOR S2448 (4), CREB S133 (5), and RSK1/2/3 S380/ $\mathrm{S} 386 / \mathrm{S} 377$ (6). NC= negative control, $\mathrm{C}=$ reference spot. (F) Crystal violet assay for cell survival following treatment with MAPK pathway specific inhibitors PD184352 (MEK1/2 inhibitor), SP600125 (broad spectrum JNK inhibitor), RWJ67657 (p38 $\alpha$ and p38 $\beta$ inhibitor, does not inhibit $\mathrm{p} 38 \gamma$ or $\delta$ ), BIRB (broad spectrum $\mathrm{p} 38$ inhibitor). $\mathrm{n}=3$. * significantly different $\mathrm{p}<0.05$. 
the ERKs, JNKs, p38s, and ERK5 [24]. Following analysis of sequencing data, many isoforms of p38 were up-regulated (MAPK11, MAPK12, MAPK13) while the $\mathrm{p} 38 \alpha$ (MAPK14) isoform was repressed. To validate observed changes in sequencing, qPCR was performed to confirm changes in MAPK signaling following enhanced miR-155 expression. Expression levels of all MAPKs (MAPK1-MAPK14) and some associated MAPKKKs (MAP3K) and MAPKKs (MAP2K7, MAP2K4, MKK4) were quantified. qPCR results demonstrate no significant change in MAPKs 1-7 (Figure 5A). A significant overall increase in expression levels of p38 isoforms $(\alpha, \delta$, and $\gamma)$ and p38 associated MAPKKS (MKK6 and MAP2K4) was observed (Figure 5B and 5D). The JNK pathway showed no significant change (Figure 5C). To determine overall changes in protein phosphorylation a protein kinase array was performed comparing the MCF-7-vector and MCF-7-miR-155 cell lines. Array results demonstrated ERK1/2 to have increased phosphorylation in the MCF7-miR-155 cell line (Figure 5E). These data suggest that miR-155 overexpression affects MAPK signaling through alterations in the p38 and ERK1/2 signaling cascades. To better determine which MAPK pathway is involved in increasing cellular proliferation, crystal violet assay was performed on MCF-7-miR-155 cells and vector following treatment of MAPK inhibitors for 72 hours. Inhibition of MEK1/2 signaling led to significant repression of cellular proliferation in the MAPK-miR-155 cell line but not the MCF-7-vector cell line, this suggest that MAPK/ERK1/2 signaling is involved in miR-155 driven proliferation (Figure 5F).

\section{AP-1 Signaling is altered in MCF-7 Breast Cancer Cells Over-Expressing miR-155}

MAPK signaling ultimately results in activation of transcription factors such as the AP-1 complex and initiates gene transcription. The p38 isoforms $\alpha, \delta$, and $\gamma$ demonstrated significant increases in expression in the miR-155 overexpressing cell lines (Figure 5B) as did the MAPK activator RAC1 and the downstream signaling molecule RSK2 (Figure 4B), all of which are known to enhance AP-1 signaling. We next sought to evaluate AP-1 complex members to determine basal level differences in Fos family members (c-Fos, FosB, Fra-1, and Fra2) and c-Jun family members (c-Jun, JunB, and JunD) through qPCR. Results demonstrate a significant increase in expression levels for Fos family members c-Fos, Fra1, and FosB and a significant repression in Fra-2 (Figure 6A). There was no change in the Jun family members c-Jun and JunB; however; JunD was significantly repressed (Figure 6B). The increase in c-Fos and Fra-1 expression also correlated with an increase in c-Fos and Fra-1 phosphorylation observed through western blot

B

C

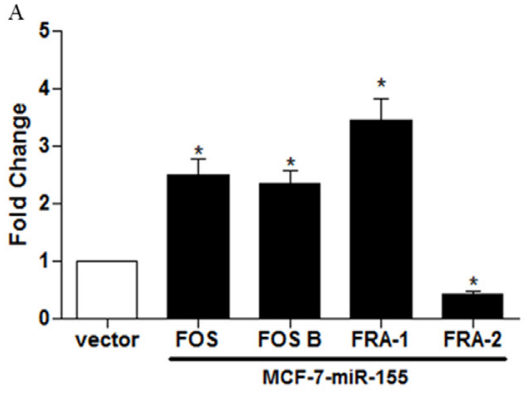

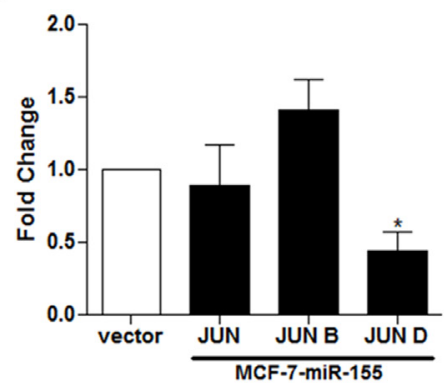

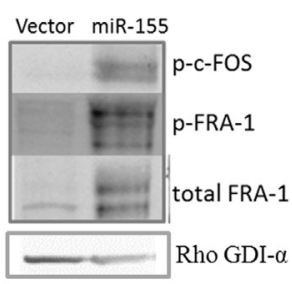

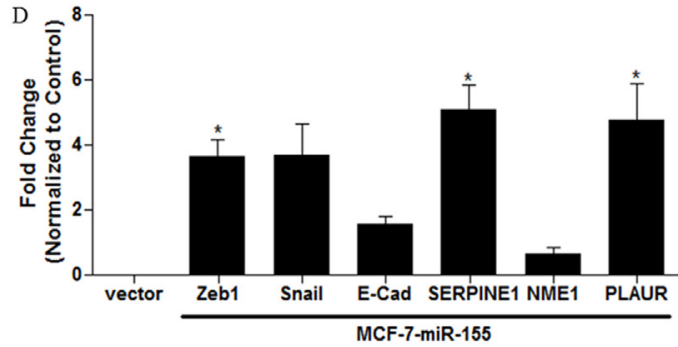

Figure 6: miR-155 Induces Changes in AP-1 Signaling .MCF-7-vector and -miR-155 cells were collected for RNA extraction and qPCR was performed to determine basal expression levels of AP-1 complex components for (A) FOS family members and (B) Jun family members, $\mathbf{n}=\mathbf{3}$. Normalization was to actin and-vector cells designated as 1 (C) Western blot analysis in MCF-7-miR-155 and -vector cells for p-c-FOS, p-c-FRA-1, total FRA-1. Normalization was to RHO GDI- $\alpha, n=3$. (D) qPCR was performed to determine basal expression levels of downstream MAPK regulated genes ZEB1, SNAIL, E-cadherin, SERPINE1, NME1, and PLAUR in MCF-7-vector cells versus -miR-155. Normalization was to actin and vector cells designated as 1 . Error bars represent $\mathrm{SEM}, * \mathrm{p}<0.05$. 
(Figure 6C). To determine if the observed increase in AP-1 gene expression and phosphorylation correlated with an increase in target gene transcription, the MAPK and AP-1 mediated genes, SerpinE1, Nme1, Plaur, Zeb1, Snail, and E-cadherin were analyzed by qPCR. Results demonstrate a significant increase in AP-1 regulated genes SerpinE1, Plaur, Zeb1, and Snail (Figure 6D).

\section{DISCUSSION}

The global effects of a miRNA can be determined by multiple factors including: the heterogeneity of the miRNA seed site, the expressed mRNA isoforms, and alterations to the mRNA 3'UTR. Here we have explored the overall effect of miR-155 expression in the MCF-7 breast cancer cell line through evaluation of the cellular transcriptome using next generation sequencing. We demonstrate that miR-155 induces increased MAPK signaling evident through both increased protein phosphorylation of MAPK signaling components (Fos, Fra-1, ERK1/2) and gene expression (Fos, Fra-1, p38). Additionally, miR-155 induced alterations in MAPK signaling genes through loss of 3'UTRs and isoform variances. Shortening of 3'UTR will result from multiple different factors such as 3'UTR methylation and alternative cleavage and polyadenylation (APA) sites giving rise to gene isoforms with truncated 3'UTRs [45-47]. Breast cancer cell lines demonstrated increased expression of 3'UTR shortening due to APA sites, which is associated with increased cellular proliferation $[45,46]$. In accordance with this, our data demonstrated loss of miR-155 targeting through shortening of mRNA 3'UTRs at the site of 8-mer seed sites and increased tumorigenesis in vivo. miR-155 is known to target genes such as SOCS1 and TP53INP1 enhancing proliferation in breast cancer cell lines [2, 48]. One intriguing idea is that the observed loss of 3'UTR and subsequent enhanced expression of MAPK associated genes is a result of miR-155 induced proliferation initiated by suppression of miR-155 targets such as SOCS1 and TP53INP1. This would result in a feed forward mechanism which propagates proliferation and tumorigenesis; however, this still remains to be fully evaluated. Evaluation of induced 3'UTR shortening is emerging as a novel layer to miRNA targeting and demonstrates how miRNA/ mRNA targeting can be cell type specific. Additionally, loss of miRNA targeting distinguishes a method for how changes in the transcriptomes can mediate the miRNA induced phenotypic profile of a cell. Loss of 3'UTR in the MAP3K10 gene are demonstrated here and previously [44]. Conversely 3'UTR shortening was not observed in the human monocytic cell line THP-1, and miR-155 was able to target the mRNA transcript in this cell line [49]. Interestingly, successful miR-155 targeting of MAP3K10 resulted in a global repression of MAPK signaling. This is reciprocal to what we show where, which is loss of miR-155 targeting of MAP3K10, increased MAP3K10 expression, and enhanced MAPK signaling. MAP3K10 alone is not the only driving factor in enhancing MAPK signaling, as we demonstrate enhanced expression of multiple MAPK signaling components. Like MAP3K10, Rac1, MAP3K14, and CEBP $\beta$ all demonstrated loss of miR-155 target site through 3'UTR shortening. In addition isoform variance was observed for the MAPK effector, RSK2. It is worth noting that the differences in mRNA 3'UTR architecture and isoform expression can influence the effects of a miRNA just as much as the miRNA itself. The inability of miRNAs to function similarly in different cellular systems can be speculated to be a result of variances in isoform transcripts and 3'UTR, drawing light on these differences aid in enhancing our knowledge of miRNA.

\section{MATERIALS AND METHODS}

\section{Cells and Reagents.}

The MCF-7 human breast cancer cell line was acquired from American Type Culture Collection (Manassas, VA). Liquid nitrogen stocks were made upon receipt and maintained until the start of study. EREluciferase and/or qPCR for ER and PgR were used to confirm sustained estrogen responsiveness of $\mathrm{ER}^{+}$cell line. Morphology and doubling times were also recorded regularly to ensure maintenance of phenotype. Cells were used for no more than 6 months after being thawed. Cells were maintained in Dulbecco's modified Eagle's medium (DMEM; pH 7.4; Invitrogen Corp., Carlsbad, CA) supplemented with 10\% fetal bovine serum (Hyclone, Salt Lake City, UT), 1\% non-essential amino acids, minimal essential amino acids, sodium pyruvate, penicillin/ streptomycin (pen/strep), and insulin under mycoplasmafree conditions at $37^{\circ} \mathrm{C}$ in humidified $5 \% \mathrm{CO} 2$ and $95 \%$ air.

\section{Generation of miR-155 cell line.}

miR-155 plasmid construct was constructed as previously described [50]. Parental MCF-7 cells were transfected with pmscv-pre-mir-155 or pmscv-vector plasmid using Lipofectamine 2000 at 1ug/ul OPTI-MEM (Invitrogen, Grand Isles, NY) as per manufacturer's protocol. Cells were grown in a $100 \mathrm{~mm}$ dish. In a $5 \mathrm{ml}$ tube, 5ug pre-mir-155 or vector plasmid was added to 100ul serum free opti-MEM then 15ul Lipofectamine was added. Following 30 minutes opti-MEM containing plasmid was added to MCF-7 cells. The following day cells were treated with $300 \mathrm{ng} / \mathrm{ml}$ puromycin. Cells were maintained in 10\% DMEM and treated with $300 \mathrm{ng} / \mathrm{ml}$ puromycin every two days for 2 weeks. Colonies were pooled and verification of mature miR-155 overexpression 
was confirmed using qPCR for mature miR-155.

\section{RNA Extraction and Quantitative Real Time RT- PCR.}

MCF-7-pmscv-vector and MCF-7-miR-155 cells were harvested and total RNA extracted using Qiagen RNeasy purification system per manufacturer's protocol (Qiagen, Valencia, CA). Quantity and quality of the RNA were determined by absorbance at 260 and $280 \mathrm{~nm}$ using the NanoDrop ND-1000. 1ug of total RNA was reversetranscribed using the iScript kit (BioRad Laboratories, Hercules, CA) and qPCR was performed using SYBRgreen (Bio-Rad Laboratories, Hercules, CA). Genes amplified, $n=4$ independent biological replicates.

\section{Crystal Violet Assay.}

MCF-7-pmscv-vector and MCF-7-miR-155 cells were grown in 10\% DMEM for 24 hours and then seeded on 48 well plates $(7,000$ cells per well) for 24 hours prior to a one time treatment with PD184352 $(5 \mu \mathrm{M})$ (Tocris, Bristol, United Kingdom), RWJ 67657 (10 $\mu \mathrm{M})$ (Johnson and Johnson Pharmaceutical Research \& Development,

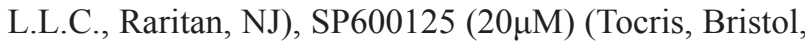
United Kingdom), BIRB $(0.5 \mu \mathrm{M})$ (Selleckchem, Houston, TX)or DMSO. After 72 hours, cells were washed once with PBS and fixed and stained using $0.1 \%$ Crystal Violet (in 20\% methanol) for 10 minutes. Cells were washed $1 \mathrm{X}$ with water and then lysed with $1 \%$ SDS. A Gene5 plate reader was used to read absorbance at wavelength 570 . Each cell line was normalized to its respective DMSO treated group, $n=3$.

\section{Western Blot Analysis.}

MCF-7-pmscv-vector and -miR-155 cells were grown in 10\% FBS DMEM. Cells were washed with PBS and lysed with M-Per lysis buffer supplemented with $1 \%$ protease inhibitor and 1\% phosphatase inhibitors (cocktail I/II) (Invitrogen, Grand Isles, NY). Supernatant containing protein extracts was obtained through centrifugation at 12,000 RPM for ten minutes at $4^{\circ} \mathrm{C}$. Protein extracted per sample was determined by absorbance at 260 and $280 \mathrm{~nm}$ using the NanoDrop ND-1000. Proteins were heat denatured and $100 \mu \mathrm{g}$ of protein were loaded per lane on Bis-Tris-nuPAGE gel (Invitrogen, Grand Isles NY). Protein was transferred to nitrocellulose through iBlot and iBlot transfer stacks as per manufacturer's protocol (Invitrogen, Grand Isles, NY). Nonspecific binding of primary antibody was blocked by incubation in 3\% milk in $1 \%$ TBS-T for 1 hour. Following blocking, membrane was incubated overnight with primary antibody for $\mathrm{p}$-c-Fos, p-Fra-1, and total Fra-1 (diluted 1:1000) (Cell Signaling
Technology, Beverly MA). The following day membrane was washed and followed by three fifteen minute washes in 1\% TBS-T. Membrane was incubated for 1 hour in secondary antibody 1:10,000 dilution (LiCor Bioscience, Lincoln NE) followed by three ten minute washes in $1 \%$ TBS-T. Band density was determined by Odyssey gel imager. Loading control protein was Rho GDI- $\alpha$ (Santa Cruz Biotechnology, Santa Cruz, CA) diluted 1:500. Experiments were conducted $n=3$ biological replicates with representative images shown.

\section{Proteome Profiler Human Phospho-Kinase Array}

MCF-7-pmscv-vector and MCF-7-miR-155 cells were seeded in $10 \mathrm{~cm} 2$ plates $2 \times 10^{6}$ and allowed to attach overnight. The next day cells were lysed according to the manufacturer's instructions (R\&D Systems Cat\# ARY003B). A single blot was used for each cell line and processed as per manufacturer's instructions with the exception being that the secondary antibody used for blots was infrared 1:10,000 dilution (LiCor Bioscience, Lincoln $\mathrm{NE}$ ). Imaging was performed on Odyssey LiCor gel imager. Individual dots were quantified using the Odyssey software and normalized using the average of positive controls as indicated on the blot.

\section{Xenograft Studies.}

4-6 wks. old ovariectomized SCID/CB17 female mice (Charles River Laboratories; Wilmington, MA) were allowed a period of adaptation in a sterile and pathogenfree environment with food and water ad libitum. Cells were harvested in the exponential growth phase using a PBS/EDTA solution and washed. Viable cells (5 x 106) in $50 \mu \mathrm{l}$ of sterile PBS suspension were mixed with 100 $\mu l$ Reduced Growth Factor Matrigel (BD Biosciences, Bedford, MA). Injections were administered into the mammary fat pad using $271 / 2$ gauge sterile syringes. Animals were divided into treatment groups of five mice each: MCF-7-pmscv-vector and MCF-7 cells transduced to overexpress miR-155. All procedures in animals were carried out under anesthesia using a mix of isofluorane and oxygen. Tumor size was measured every 2-3 days using digital calipers. The volume of the tumor was calculated using the formula: $4 / 3 \pi \mathrm{LS} 2$ ( $\mathrm{L}=$ larger radius; $\mathrm{S}=$ shorter radius). At necropsy, animals were euthanized by cervical dislocation after exposure to $\mathrm{CO}^{2}$. Tumors were removed and frozen in liquid nitrogen or fixed in $10 \%$ formalin for further analysis. All procedures involving these animals were conducted in compliance with State and Federal laws, standards of the U.S. Department of Health and Human Services, and guidelines established by Tulane University Animal Care and Use Committee. The facilities and laboratory animals program of Tulane University are accredited by the Association for the Assessment and 
Accreditation of Laboratory Animal Care.

\section{Next Generation Sequencing.}

Full methods and parameters of alignment were performed as previously described [44]. Briefly, MCF7-pmscv-vector and -miR-155 cell lines were harvested for total RNA extraction using the mRNeasy (Qiagen) kit. Next Generation Sequencing (NGS) was performed using the Illumina Genome Analyzer II. Results were aligned in the fastq format against the annotated human reference genome (Hg19). Reads per kilobase of exon model per million mapped reads (RPKM) were generated through SAMMate which compiled alignments generated with the Novoalign (http://www.novocraft.com) and TopHat aligners. Use of TopHat allowed for mapping of exonexon junctions that may have initially read as unmapped reads [51]. Fold change in gene expression was calculated using RPKM. Gene expression analysis was viewed in Integrative Genomics Viewer (IGV).

\section{Statistical Analysis.}

Statistical Analysis was performed using Graph Pad Prism 5. Student's t test was used to determine $\mathrm{p}$ values and statistically significant values had a p-values of $<0.05$.

\section{Grant Support:}

This research was supported by the National Institutes of Health - CA125806 and the Office of Naval Research N00014-16-1-1136 (ME Burow), the National Center for Research Resources P20RR020152 and The Department of Defense Breast Cancer Research Program BC085426 (BM Collins-Burow), the National Institutes of health - R01AI101046, R01AI106676 and R01CA138268 (EK Flemington), and grants from the National Center for Research Resources (5P20RR020152-09) and the National Institute of General Medical Sciences (8 P20 GM10351809 ) from the National Institutes of Health.

\section{Conflict of Interest:}

None declared

\section{REFERENCES}

1. Calin GA, Croce CM: MicroRNA signatures in human cancers. Nature reviews Cancer 2006, 6:857-866.

2. Jiang S, Zhang HW, Lu MH, He XH, Li Y, Gu H, Liu MF, Wang ED: MicroRNA-155 functions as an OncomiR in breast cancer by targeting the suppressor of cytokine signaling 1 gene. Cancer research 2010, 70:3119-3127.
3. Kong W, He L, Coppola M, Guo J, Esposito NN, Coppola D, Cheng JQ: MicroRNA-155 regulates cell survival, growth, and chemosensitivity by targeting FOXO3a in breast cancer. The Journal of biological chemistry 2010, 285:17869-17879.

4. Zhao JJ, Lin J, Yang H, Kong W, He L, Ma X, Coppola D, Cheng JQ: MicroRNA-221/222 negatively regulates estrogen receptor alpha and is associated with tamoxifen resistance in breast cancer. The Journal of biological chemistry 2008, 283:31079-31086.

5. Riaz M, van Jaarsveld MT, Hollestelle A, Prager-van der Smissen WJ, Heine AA, Boersma AW, Liu J, Helmijr J, Ozturk B, Smid M, et al: miRNA expression profiling of 51 human breast cancer cell lines reveals subtype and driver mutation-specific miRNAs. Breast cancer research : BCR 2013, 15:R33

6. Zhu J, Zheng Z, Wang J, Sun J, Wang P, Cheng X, Fu L, Zhang L, Wang Z, Li Z: Different miRNA expression profiles between human breast cancer tumors and serum. Frontiers in genetics 2014, 5:149.

7. Singh R, Mo YY: Role of microRNAs in breast cancer. Cancer biology \& therapy 2013, 14:201-212.

8. Lu Z, Ye Y, Jiao D, Qiao J, Cui S, Liu Z: miR-155 and miR31 are differentially expressed in breast cancer patients and are correlated with the estrogen receptor and progesterone receptor status. Oncology letters 2012, 4:1027-1032.

9. Wang F, Zheng Z, Guo J, Ding X: Correlation and quantitation of microRNA aberrant expression in tissues and sera from patients with breast tumor. Gynecologic oncology 2010, 119:586-593.

10. Ling N, Gu J, Lei Z, Li M, Zhao J, Zhang HT, Li X: microRNA-155 regulates cell proliferation and invasion by targeting FOXO3a in glioma. Oncology reports 2013, 30:2111-2118

11. Zhang C, Zhao J, Deng H: 17beta-estradiol up-regulates miR-155 expression and reduces TP53INP1 expression in MCF-7 breast cancer cells. Molecular and cellular biochemistry 2013, 379:201-211.

12. Tam W, Ben-Yehuda D, Hayward WS: bic, a novel gene activated by proviral insertions in avian leukosis virusinduced lymphomas, is likely to function through its noncoding RNA. Molecular and cellular biology 1997, 17:1490-1502.

13. Faraoni I, Antonetti FR, Cardone J, Bonmassar E: miR-155 gene: a typical multifunctional microRNA. Biochimica et biophysica acta 2009, 1792:497-505.

14. Costinean S, Zanesi N, Pekarsky Y, Tili E, Volinia S, Heerema N, Croce CM: Pre-B cell proliferation and lymphoblastic leukemia/high-grade lymphoma in $\mathrm{E}(\mathrm{mu})$ miR155 transgenic mice. Proceedings of the National Academy of Sciences of the United States of America 2006, 103:7024-7029.

15. Czyzyk-Krzeska MF, Zhang X: MiR-155 at the heart of oncogenic pathways. Oncogene 2014, 33:677-678. 
16. O'Connell RM, Chaudhuri AA, Rao DS, Baltimore D: Inositol phosphatase SHIP1 is a primary target of miR-155. Proceedings of the National Academy of Sciences of the United States of America 2009, 106:7113-7118.

17. Trotta R, Chen L, Costinean S, Josyula S, Mundy-Bosse BL, Ciarlariello D, Mao C, Briercheck EL, McConnell KK, Mishra A, et al: Overexpression of miR-155 causes expansion, arrest in terminal differentiation and functional activation of mouse natural killer cells. Blood 2013, 121:3126-3134.

18. Thai TH, Patterson HC, Pham DH, Kis-Toth K, Kaminski DA, Tsokos GC: Deletion of microRNA-155 reduces autoantibody responses and alleviates lupus-like disease in the Fas(lpr) mouse. Proceedings of the National Academy of Sciences of the United States of America 2013, 110:20194-20199.

19. Jeansonne D, Pacifici M, Lassak A, Reiss K, Russo G, Zabaleta J, Peruzzi F: Differential Effects of MicroRNAs on Glioblastoma Growth and Migration. Genes 2013, 4:4664.

20. Marsolier J, Pineau S, Medjkane S, Perichon M, Yin Q, Flemington E, Weitzman MD, Weitzman JB: OncomiR addiction is generated by a miR-155 feedback loop in Theileria-transformed leukocytes. PLoS pathogens 2013, 9:e1003222.

21. Fahmi A, Smart N, Punn A, Jabr R, Marber M, Heads R: p42/p44-MAPK and PI3K are sufficient for IL-6 family cytokines/gp130 to signal to hypertrophy and survival in cardiomyocytes in the absence of JAK/STAT activation. Cellular signalling 2013, 25:898-909.

22. Robinson MJ, Cobb MH: Mitogen-activated protein kinase pathways. Current opinion in cell biology 1997, 9:180-186.

23. Roux PP, Blenis J: ERK and p38 MAPK-activated protein kinases: a family of protein kinases with diverse biological functions. Microbiology and molecular biology reviews : MMBR 2004, 68:320-344.

24. Boulton TG, Nye SH, Robbins DJ, Ip NY, Radziejewska E, Morgenbesser SD, DePinho RA, Panayotatos N, Cobb MH, Yancopoulos GD: ERKs: a family of proteinserine/threonine kinases that are activated and tyrosine phosphorylated in response to insulin and NGF. Cell 1991, 65:663-675.

25. Kant S, Schumacher S, Singh MK, Kispert A, Kotlyarov A, Gaestel M: Characterization of the atypical MAPK ERK4 and its activation of the MAPK-activated protein kinase MK5. The Journal of biological chemistry 2006, 281:35511-35519.

26. Cargnello M, Roux PP: Activation and function of the MAPKs and their substrates, the MAPK-activated protein kinases. Microbiology and molecular biology reviews : MMBR 2011, 75:50-83.

27. Nishimoto S, Nishida E: MAPK signalling: ERK5 versus ERK1/2. EMBO reports 2006, 7:782-786.

28. Gilley R, March HN, Cook SJ: ERK1/2, but not ERK5, is necessary and sufficient for phosphorylation and activation of c-Fos. Cellular signalling 2009, 21:969-977.

29. Chung J, Uchida E, Grammer TC, Blenis J: STAT3 serine phosphorylation by ERK-dependent and -independent pathways negatively modulates its tyrosine phosphorylation. Molecular and cellular biology 1997, 17:6508-6516.

30. Marampon F, Ciccarelli C, Zani BM: Down-regulation of c-Myc following MEK/ERK inhibition halts the expression of malignant phenotype in rhabdomyosarcoma and in non muscle-derived human tumors. Molecular cancer 2006, 5:31.

31. Mebratu Y, Tesfaigzi Y: How ERK1/2 activation controls cell proliferation and cell death: Is subcellular localization the answer? Cell cycle 2009, 8:1168-1175.

32. Jiang Y, Chen C, Li Z, Guo W, Gegner JA, Lin S, Han $\mathrm{J}$ : Characterization of the structure and function of a new mitogen-activated protein kinase (p38beta). The Journal of biological chemistry 1996, 271:17920-17926.

33. Jiang Y, Gram H, Zhao M, New L, Gu J, Feng L, Di Padova F, Ulevitch RJ, Han J: Characterization of the structure and function of the fourth member of $\mathrm{p} 38$ group mitogen-activated protein kinases, p38delta. The Journal of biological chemistry 1997, 272:30122-30128.

34. Risco A, Cuenda A: New Insights into the p38gamma and p38delta MAPK Pathways. Journal of signal transduction 2012, 2012:520289.

35. Price MA, Cruzalegui FH, Treisman R: The p38 and ERK MAP kinase pathways cooperate to activate Ternary Complex Factors and c-fos transcription in response to UV light. The EMBO journal 1996, 15:6552-6563.

36. Enslen H, Raingeaud J, Davis RJ: Selective activation of p38 mitogen-activated protein (MAP) kinase isoforms by the MAP kinase kinases MKK3 and MKK6. The Journal of biological chemistry 1998, 273:1741-1748.

37. Raingeaud J, Whitmarsh AJ, Barrett T, Derijard B, Davis RJ: MKK3- and MKK6-regulated gene expression is mediated by the $\mathrm{p} 38$ mitogen-activated protein kinase signal transduction pathway. Molecular and cellular biology 1996, 16:1247-1255.

38. Pramanik R, Qi X, Borowicz S, Choubey D, Schultz RM, Han J, Chen G: p38 isoforms have opposite effects on AP-1-dependent transcription through regulation of c-Jun. The determinant roles of the isoforms in the p38 MAPK signal specificity. The Journal of biological chemistry 2003, 278:4831-4839.

39. Chen W, Bowden GT: Activation of p38 MAP kinase and ERK are required for ultraviolet-B induced c-fos gene expression in human keratinocytes. Oncogene 1999, 18:7469-7476.

40. Pottier N, Maurin T, Chevalier B, Puissegur MP, Lebrigand $\mathrm{K}$, Robbe-Sermesant $\mathrm{K}$, Bertero $\mathrm{T}$, Lino Cardenas CL, Courcot E, Rios G, et al: Identification of keratinocyte growth factor as a target of microRNA-155 in lung fibroblasts: implication in epithelial-mesenchymal 
interactions. PloS one 2009, 4:e6718.

41. Johansson J, Berg T, Kurzejamska E, Pang MF, Tabor V, Jansson M, Roswall P, Pietras K, Sund M, Religa P, Fuxe J: MiR-155-mediated loss of C/EBPbeta shifts the TGF-beta response from growth inhibition to epithelialmesenchymal transition, invasion and metastasis in breast cancer. Oncogene 2013, 32:5614-5624.

42. Sandberg R, Neilson JR, Sarma A, Sharp PA, Burge CB: Proliferating cells express mRNAs with shortened 3' untranslated regions and fewer microRNA target sites. Science 2008, 320:1643-1647.

43. Akman BH, Can T, Erson-Bensan AE: Estrogen-induced upregulation and 3'-UTR shortening of CDC6. Nucleic acids research 2012, 40:10679-10688.

44. Xu G, Fewell C, Taylor C, Deng N, Hedges D, Wang X, Zhang K, Lacey M, Zhang H, Yin Q, et al: Transcriptome and targetome analysis in MIR155 expressing cells using RNA-seq. Rna 2010, 16:1610-1622.

45. Elkon R, Drost J, van Haaften G, Jenal M, Schrier M, Oude Vrielink JA, Agami R: E2F mediates enhanced alternative polyadenylation in proliferation. Genome biology 2012, 13:R59.

46. Mayr C, Bartel DP: Widespread shortening of 3'UTRs by alternative cleavage and polyadenylation activates oncogenes in cancer cells. Cell 2009, 138:673-684.
47. Meyer KD, Saletore Y, Zumbo P, Elemento O, Mason CE, Jaffrey SR: Comprehensive analysis of mRNA methylation reveals enrichment in 3' UTRs and near stop codons. Cell 2012, 149:1635-1646.

48. Zhang CM, Zhao J, Deng HY: MiR-155 promotes proliferation of human breast cancer MCF-7 cells through targeting tumor protein 53-induced nuclear protein 1 . Journal of biomedical science 2013, 20:79.

49. Zhu J, Chen T, Yang L, Li Z, Wong MM, Zheng X, Pan X, Zhang L, Yan H: Regulation of microRNA-155 in atherosclerotic inflammatory responses by targeting MAP3K10. PloS one 2012, 7:e46551.

50. Yin Q, McBride J, Fewell C, Lacey M, Wang X, Lin Z, Cameron J, Flemington EK: MicroRNA-155 is an EpsteinBarr virus-induced gene that modulates Epstein-Barr virusregulated gene expression pathways. Journal of virology 2008, 82:5295-5306.

51. Trapnell C, Pachter L, Salzberg SL: TopHat: discovering splice junctions with RNA-Seq. Bioinformatics 2009, 25:1105-1111.

52. Martin EC, Rhodes LV, Elliott S, Krebs AE, Nephew KP, Flemington EK, Collins-Burow BM, Burow ME. microRNA regulation of mammalian target of rapamycin expression and activity controls estrogen receptor function and RAD001 sensitivity. Molecular Cancer, in press 